\title{
Identification of Candidate Cerebrospinal Fluid Biomarkers in Parkinsonism Using Quantitative Proteomics
}

Magdalinou, NK ${ }^{1}$, M.D., Noyce AJ ${ }^{1}$, M.D., Pinto R2, D.I., Lindstrom E ${ }^{3}$, M.Sc., Holmén-Larsson J3, Ph.D., Holtta $\mathrm{M}^{3}$, Ph.D., Blennow K3 ${ }^{3}$, M.D., Ph.D., Morris HR ${ }^{4}$, M.D., Ph.D., Skillbäck, T³ ${ }^{3}$ M.D., Warner TT ${ }^{1}$, M.D., Ph.D., Lees AJ ${ }^{1}$, M.D., Ph.D., Pike $\mathrm{I}^{5}$, Ph.D., Ward $\mathrm{M}^{5}$, Ph.D., Zetterberg H${ }^{3,6}$, M.D., Ph.D., Gobom J ${ }^{3}$, Ph.D.

${ }^{1}$ Reta Lila Weston Institute of Neurological Studies, UCL Institute of Neurology, Queen Square, London, UK

2Institute of Chemistry University of Umeå, Umeå Sweden

${ }^{3}$ Clinical Neurochemistry Laboratory, Institute of Neuroscience and Physiology, the Sahlgrenska Academy at the University of Gothenburg, Mölndal, Sweden

${ }^{4}$ Department of Clinical Neuroscience, UCL Institute of Neurology, Royal Free Hospital, London, UK

${ }^{5}$ Proteome Sciences plc, London, United Kingdom

${ }^{6}$ Department of Molecular Neuroscience, UCL Institute of Neurology, Queen Square, London, UK

\section{Corresponding Author:}

Nadia Magdalinou

Reta Lila Weston Institute

1 Wakefield Street

London

WC1N 1PJ

Phone: +44 2076794246

Email: n.magdalinou@ucl.ac.uk

Word Count: 2975

\section{References 32}

Keywords: Parkinson's disease, Atypical parkinsonian syndrome, Biomarkers, Proteomics 


\section{Abstract}

Introduction Neurodegenerative parkinsonian syndromes have significant clinical and pathological overlap, making early diagnosis difficult. Cerebrospinal fluid (CSF) biomarkers may aid the differentiation of these disorders, but other than $\alpha$-synuclein and neurofilament light chain protein, which have limited diagnostic power, specific protein biomarkers remain elusive.

Objectives To study disease mechanisms and identify possible CSF diagnostic biomarkers through discovery proteomics, which discriminate parkinsonian syndromes from healthy controls.

Methods CSF was collected consecutively from 134 participants; Parkinson's disease $(n=26)$, atypical parkinsonian syndromes $(n=78$, including progressive supranuclear palsy $(\mathrm{n}=36)$, multiple system atrophy $(\mathrm{n}=28)$, corticobasal syndrome ( $n=14)$ ), and elderly healthy controls $(n=30)$. Participants were divided into a discovery and a validation set for analysis. The samples were subjected to tryptic digestion, followed by liquid chromatography-mass spectrometry analysis for identification and relative quantification by isobaric labelling. Candidate protein biomarkers were identified based on the relative abundances of the identified tryptic peptides. Their predictive performance was evaluated by analysis of the validation set.

Results 79 tryptic peptides, derived from 26 proteins were found to differ significantly between atypical parkinsonism patients and controls. They included acute phase/inflammatory markers and neuronal/synaptic markers, which were respectively increased or decreased in atypical parkinsonism, while their levels in PD subjects were intermediate between controls and atypical parkinsonism. 
Conclusion Using an unbiased proteomic approach, proteins were identified that were able to differentiate atypical parkinsonian syndrome patients from healthy controls. Our study indicates that markers that may reflect neuronal function and/or plasticity, such as the amyloid precursor protein, and inflammatory markers may hold future promise as candidate biomarkers in parkinsonism.

\section{Keywords}

Parkinsonian disorders, Parkinson's disease, progressive supranuclear palsy, multiple system atrophy, corticobasal syndrome, biomarker, proteomics, cerebrospinal fluid, isobaric labelling 


\section{Introduction}

Parkinson's disease (PD) is the most common neurodegenerative movement disorder and the number of patients is expected to double over the next two decades, presenting a huge social and economic challenge [1]. Atypical parkinsonian syndromes, such as progressive supranuclear palsy (PSP), multiple system atrophy (MSA) and corticobasal syndrome (CBS) represent rarer but more aggressive forms of parkinsonism. Atypical parkinsonian syndromes and PD often present in a strikingly similar manner, making an accurate early diagnosis difficult. Despite similar clinical characteristics, these diseases differ substantially in their prognosis, pathological features and therapeutic response[2,3]. There is an urgent need to identify biomarkers for parkinsonian disorders to enable earlier, accurate diagnosis, monitor disease progression and response to drug therapies.

Cerebrospinal fluid (CSF) has been widely investigated in parkinsonian disorders and may serve as a source of biomarkers that reflect brain-related disease processes. In PD, clinical studies of candidate proteins, such as $\alpha$-synuclein and neurofilament light chain protein, show minimal changes and high assay variability and have thus far not resulted in a diagnostically useful biomarker (for a review see[4]).

To date studies employing unbiased proteomic approaches have led to the identification of various combinations of CSF proteins that differ in abundance between patient groups[5-9], but singling out promising candidate markers among the many detected proteins has proven challenging. Part of the problem is the disproportionality of the published proteomic data sets, which contain many identified proteins but only few patients, leading to a risk of over-fitting 
statistical models. Other complicating factors include study groups with heterogeneous disease characteristics and experimental variation. In order to overcome these limitations, studies of larger patient groups are required. While previously limited by the long analysis times of liquid chromatography-mass spectrometry (LC-MS) used in discovery proteomics workflows, performing large studies is now possible through the development of timesaving multiplex isobaric labeling techniques, such as tandem mass tags (TMT)[10].

In this study, we used multiplex isobaric labeling to perform the largest proteomic study of parkinsonism to date, and the first study to include several atypical parkinsonian syndromes and healthy controls. Our aim was to shed light on potential disease mechanisms and explore the possibility of new diagnostic markers.

\section{Methods}

\section{Study Participants}

This is a cross-sectional study of patients with parkinsonian disorders and healthy controls. Participants were prospectively enrolled over a two-year period from 2011 to 2013 from the movement disorders, cognitive and autonomic disorders clinics at the National Hospital for Neurology and Neurosurgery, Queen Square, London. The diagnoses of probable PD, PSP, CBS and MSA were based on consensus operational criteria[3, 11-13]. Patients included in the study were 40-85 years old and under follow-up for at least two years. The clinical diagnosis was corroborated by at least two neurologists with experience in movement disorders (AJL, TTW, HRM, AJN, NM). 
Further information patient characterization and exclusion criteria are listed in "Supplementary Materials - Methods".

\section{Ethics Approval}

The study was conducted in accordance with local clinical research regulations and an informed consent was obtained from all subjects, including access to clinical data and imaging. The study was performed in accordance with the provisions of the Helsinki declaration and the research protocol was approved by the London Queen Square research ethics committee.

\section{CSF Collection and Storage}

We adhered to a standardized protocol for the collection and storage of CSF as recommended by the Alzheimer's Association QC Program for AD CSF biomarkers (www.neurochem.gu.se/TheAlzAssQCProgram). Details on the procedure are given in "Supplementary Materials - Methods".

\section{Experimental Design}

136 subjects were included in the analysis. Subjects were randomly divided into a discovery (13 PD, 39 atypical parkinsonian patients and 15 healthy controls) and a validation (13 PD, 43 atypical parkinsonian patients and 13 healthy controls) set. For demographic and clinical characteristics see Table 1. CSF samples from the two sets were prepared and analyzed separately. Details of CSF sample preparation are provided in "Supplementary Materials- Methods".

\section{MS analysis}

The samples were reconstituted in a solution of $2 \%$ acetonitrile, $0.1 \%$ TFA (600 $\mu \mathrm{l}$ ). Aliquots of $2 \mu \mathrm{l}$ were analyzed with a nano-LC (Ultimate 3000, Thermo Scientific) equipped with a $\mathrm{C}_{18}$ trap column (PepMap Acclaim $75 \mu \mathrm{m} * 20 \mathrm{~mm}$, 
Thermo Scientific), and a $C_{18}$ separation column (PepMap Acclaim $75 \mu \mathrm{m} * 500$ $\mathrm{mm}$, Thermo Scientific), coupled to a Q-Exactive electrospray ionization mass spectrometer (Thermo Scientific), fitted with a FlexiSpray ion source. The loading buffer was $2 \%$ acetonitrile, $0.05 \%$ TFA; Buffer A was $0.1 \%$ formic acid; and Buffer B was $84 \%$ acetonitrile, $0.1 \%$ formic acid. The following gradient was used: $\mathrm{t}=0 \mathrm{~min}, \mathrm{~B}=3 \% ; 140 \min , \mathrm{B}=30 \% ; 160 \mathrm{~min}, \mathrm{~B}=45 \% ; 165 \mathrm{~min}, \mathrm{~B}=80 \%$. The mass spectrometer was operated in the positive ion mode. Data-dependent acquisition was used, acquiring one full MS scan (R=70k, AGC target=1e6, max $\mathrm{IT}=250 \mathrm{~ms})$ and up to 10 consecutive MS/MS scans $(\mathrm{R}=17.5 \mathrm{k}$, AGC target=5e4, $\max \mathrm{IT}=60 \mathrm{~ms}$ ). Data processing was performed within the software ProteomeDiscoverer 1.4 (Thermo Scientific), using Mascot (MatrixScience) for protein identification (precursor $\Delta \mathrm{m}$ tolerance $=5 \mathrm{ppm}$, fragment $\Delta \mathrm{m}$ tolerance $=20$ milli mass units, missed cleavages $=2$, fixed modifications $=$ carbamidomethylation, variable modifications=oxidation of methionine), searching the human subset of the UniProtKB Swiss-Prot database (release 13-10) (www.uniprot.org). Percolator (MatrixScience) was used for scoring peptide specific matches, and 1\% false discovery rate (FDR) was set as threshold for identification. The following settings were used for reporter ion quantification: Integration tolerance $=150 \mathrm{ppm}$; Integration Method= Most Confident Centroid; exclusion of MS/MS spectra > $50 \%$ co-isolation; normalize on protein median.

\section{Data analysis}

Statistical data analysis was performed using R Statistics, with the libraries pROC[14] and ggplot2[15]. T-tests were performed on log-transformed TMT reporter ion ratios of peptides detected in $>50 \%$ of the samples to assess the 
significance of differences in the relative abundance of the peptides between the patient groups and the controls in the discovery set. The p-values were corrected for multiple testing according to Benjamini-Hochberg[16]. Peptides with $\mathrm{p}<0.05$ were considered significant. These were evaluated in the validation set, applying the same methods and criteria as for the discovery set, considering, for multiple testing correction, the t-tests performed in the validation set. Spearman's rank correlation coefficient was used to evaluate the correlation between the identified biomarker candidates and age.

\section{Results}

Demographic characteristics of the participants in the study are given in Table 1. Mass spectrometric analysis of the discovery set identified 5043 tryptic peptides, out of which 1689 were detected in $>50 \%$ of the participants. Ninety peptides had significantly $(\mathrm{p}<0.05)$ different abundances in atypical parkinsonian syndromes versus healthy controls. Out of these, 89 were also detected in $>50 \%$ of the patients in the validation set. In the validation set, 80 out of the 89 peptides differed significantly $(\mathrm{p}<0.05)$ in abundance between atypical parkinsonian syndromes and healthy controls. Between the two sets, all 80 differed in the same direction. The median difference in percentage points for the peptides between the two sets was 5\% (max. 42\%, min. 1\%). The relative abundance of one peptide, belonging to complement component $\mathrm{C}$, correlated significantly with age $(\mathrm{p}=0.0074)$, and was therefore disqualified as a candidate biomarker. In total, 79 tryptic peptides, belonging to 26 proteins were identified as candidate biomarkers of atypical parkinsonian syndromes (Supplementary Table s1, Figure 1). 


\section{Pathologically confirmed group}

Since the study began 11 patients have died and donated their brains for pathological examination. Six PSP, three MSA and two CBS patients were diagnosed according to standard pathological criteria. Two of the eleven patients were misclassified during life (one patient was clinically diagnosed with PSP and had CBS pathology, and one was clinically diagnosed with MSA and had PSP pathology).

\section{Discussion}

The 26 proteins identified that differed between healthy controls and patients with atypical parkinsonian syndromes fit into three categories: acute phase/inflammatory (which were significantly increased in atypical parkinsonian syndromes) and neuronal/synaptic proteins or proteins involved in cancer/metastasis formation (which were significantly decreased in atypical parkinsonian syndromes compared with healthy controls) (Table 2). For all proteins, the levels in PD were intermediate between healthy controls and atypical parkinsonian syndromes, which may reflect the slower and less widespread neurodegeneration in PD compared to more aggressive forms of parkinsonism. This trend was strongest for the acute phase/inflammatory proteins, whereas among the neuronal/synaptic proteins there were several that are more specific to atypical parkinsonian syndromes. 13 proteins had AUC values between 0.70 and 0.80 , which is similar to earlier reported AUCs for CSF neurofilament light chain protein and better than what can be achieved using CSF $\alpha$-synuclein $[17,18]$. 


\section{Description of Proteins Identified and Previous CSF Neurodegenerative}

\section{Studies}

A summary of the known functions of each protein identified is given in Table S2 in Supplementary Material online. A literature review revealed that 22 out of those 26 proteins have already been described in previous CSF studies in neurodegenerative diseases. 16 proteins were previously identified in studies involving parkinsonian groups and 15 proteins were previously identified in proteomic studies. Eight proteins have been found in published proteomics studies involving parkinsonian groups.

\section{Acute phase/Inflammatory Proteins}

Neuroinflammation is a critical component in the progression of many neurodegenerative diseases. We reproduced published results showing alteration in CSF proteome of parkinsonian patients for proteins involved in immune response.

The complement system consists of over 30 proteins and is an important member of both the innate and adaptive immune systems[19]. Inappropriate activation of complement can contribute to disease. A proteomics study investigated serum taken from patients with $\mathrm{PD}$, amyotrophic lateral sclerosis (ALS), neurological and healthy controls[20]. PD and ALS patients showed a marked increase in seven out of nine complement related proteins. This is consistent with what was demonstrated in our study where atypical parkinsonian patients had significantly increased CSF levels of complements C9 and factor H compared with healthy controls. A recent study from our group using a commercially available enzyme-linked immunosorbent assay (ELISA)[17] showed increased CSF levels of YKL-40 in 
atypical parkinsonian syndromes (especially PSP), with lowest levels in healthy controls (PD levels were slightly higher than controls). In our current study, using a mass-spectrometry approach, we find further supportive evidence for elevated YLK-40 in atypical parkinsonian syndromes.

CSF $\alpha$-1-antichymotrypsin (ACT) levels have been reported as being significantly increased in MSA compared with neurological controls[21]. Here we also report significantly increased CSF ACT levels in atypical parkinsonian patients compared with healthy controls.

\section{Neuronal/Synaptic Proteins}

Recently, secretogranin2 gene was established as a signal integrator of glutamate and dopamine inputs[22]. A previous proteomics study has demonstrated that using two secretogranin 1 fragments and two other markers, PD and healthy controls could be differentiated from atypical parkinsonian patients[6]. We have replicated these findings by showing significantly reduced CSF secretogranin 1-3 levels in atypical parkinsonian subjects, differentiating them from healthy controls.

In another proteomics study, CSF neuronal pentraxin receptor (NPR) was found to be significantly decreased in PD compared with healthy controls[23]. We also found reduced CSF NPR levels in PD compared with healthy controls, but the most significant decrease was again in the atypical parkinsonian group. Our data showed reduced CSF levels of all proteins associated with APP in atypical parkinsonian patients compared with healthy controls. This is consistent with our recent findings using commercially available ELISAs[17], which demonstrated significantly decreased levels of soluble APP $\alpha$ and $\beta$ in atypical parkinsonian patients compared with PD and healthy controls. Amyloid- 
like protein 1 (APLP1) has a probable role in synaptogenesis or synaptic maturation[24]. In a recent proteomics study, CSF APLP1 was found to be significantly reduced in PD compared with patients with Alzheimer's disease and healthy controls[25]. Calsyntenin-1 contributes to the axonal transport of APP. Using proteomics technology, CSF calsyntenin 1 (isoform 2) was found to be decreased in PD compared with healthy controls[23].

The reason for the APP reduction is unclear but it is interesting to note that APP is bound to the mitochondrial outer membrane and has been implicated in mitochondrial dysfunction, which may contribute to some neurodegenerative diseases[26].

Decreased levels of somatostatin have been found in CSF of parkinsonian patients[27] compared with AD patients and elderly controls, which is in accordance with our data. In another proteomics study, CSF neuronal cell adhesion molecule was found to be decreased in PD compared with healthy controls[23]. Even though the most significant decrease in neuronal cell adhesion molecule levels in our study was in atypical parkinsonian syndromes, PD levels were also lower compared to healthy controls.

\section{Proteins associated with cancer/metastasis formation}

The pathogenesis of both neurodegenerative and carcinogenic diseases is due to a constellation of genetic and environmental factors. Causative genes have been found to be involved in both processes usually showing an inverse correlation between the risk of developing cancer and a neurodegenerative disorder, especially PD[28]. Many of the genes associated with cancer and neurodegeneration have a central role in cell cycle control, DNA repair and kinase signaling[28].In addition, post-translation modifications play an 
important role in both cancer and neurodegeneration. For example, loss of function of Parkin (PARK2) gene leads to PD[29] and Parkin in cancer acts as tumour suppressor.[30] In our study, we have found four proteins associated with cancer/metastasis formation, the precursor of one of which, FAM3C, has already been identified in another proteomics study as a potential CSF biomarker in dementia with Lewy bodies (DLB)[5]- a synucleinopathy closely linked to PD.

\section{Genome-Wide Association (GWA) studies}

Recent observations from GWA studies of PSP patients compared to controls provide further insight at a genomic level[31]. For PSP, genome wide significance was found for single nucleotide polymorphisms (SNPs) in the region of the MAPT gene (aggregated tau as neurofibrillary tangles and coiled bodies are pathological hallmark of PSP), and in the regions of STX6 (involved in synaptic transmission and vesicular trafficking) and EIF2AK3 (which localizes to the endoplasmic reticulum and is involved in the unfolded protein response). Significance was also reported in the region of MOBP gene (which has a less well defined role but relates to either myelin or oligodendrocytes). Our finding of significant change in a number of synaptic proteins in the atypical parkinsonian syndromes aligns with mounting evidence of pathways in vesicular transport and synaptic transmission being vital in the pathogenesis of PSP.

\section{Differentiation between PD and APS}

To assess the diagnostic potential of the identified candidate markers, we performed ROC curve analysis between the disease groups. We found five proteins with AUC values between 0.73 and 0.80 differentiating between PD and atypical parkinsonian groups. They included $\alpha$-1-antichymotrypsin, 
secretogranin 1-3, endothelin B receptor like protein 2 and neuronal pentraxin receptor. These predominantly neuronal/synaptic proteins could be promising diagnostic biomarkers.

\section{Strengths}

This is the most comprehensive proteomic study of parkinsonian syndromes to date. The study design is robust as both a discovery and validation set were used and analyzed separately. Randomly splitting the original sample of subjects, using one database as a training set to create the predictor and the remainder of subjects for validation is used in order to overcome the common proteomics studies' problem of overfitting the model[32]. Our diseased groups are well defined and were followed up for at least two years after inclusion into the study, improving the accuracy of the clinical diagnoses. For $13 \%$ of the atypical parkinsonian patients, there is pathological confirmation. The number of identified proteins was considerably higher compared to the other proteomics study in parkinsonian conditions. The statistical data analysis method employed was unbiased and all 26 candidate biomarkers identified in atypical parkinsonian syndromes vs. healthy controls can be interpreted within the framework of neurodegeneration. 22 out of those 26 proteins have already been described in previous CSF neurodegenerative studies and 15 proteins were identified in studies also using proteomics technology. Thus we have found significant overlap with previous CSF proteomics studies in neurodegenerative diseases.

Abdi et al compared relative changes in the CSF proteome among with $10 \mathrm{AD}, 10$ PD and 5 DLB compared with 10 healthy controls. They found 136 proteins uniquely associated with AD, 72 with PD and 101 with DLB[5]. However, this 
study included small numbers of patients and lacked a validation group. Nevertheless, there was overlap with six of the proteins identified in our study: neuronal pantraxin 1, APP, APLP2, FAM3C, neuroblastoma suppressor of tumerigenicity 1 and probable G-protein coupled receptor 158.

To our knowledge, Constantinescu's study[6] is the only proteomics study in the literature with comparable diseased groups to ours (PD $n=56, \operatorname{MSA} n=42$, PSP $n=36, C B D n=9$ ) and 24 healthy controls. They concluded that a CSF proteomic profile consisting of four proteins (ubiquitin, $\beta 2$-microglobulin and two secretogranin 1 fragments) could differentiate PD and healthy controls from atypical parkinsonian patients with an AUC of 0.8. Constantinescu et al used profiling based methods that lack the dynamic range to detect differences other than those seen for relatively abundant proteins. Nevertheless, there was an overlap with one protein identified in our study- secretogranin 1. Constantinescu et al could not differentiate between PD patients and controls. In our study, using more advanced proteomics technology, we were able to show some degree of differentiation between PD and healthy controls, even though the greatest discrimination was still between atypical parkinsonian patients and healthy controls.

\section{Limitations}

A recurring challenge in the study of neurodegenerative disorders is clinical misdiagnosis of patients. In our pathologically confirmed patient group, two out of 11 atypical parkinsonian patients were misdiagnosed during life. Another challenge is controls, who are healthy at the inclusion of the study, but may 
develop neurodegenerative conditions that become clinically apparent years later. In our study, there were aberrant values for several peptides from the same healthy control. Within two years of inclusion in the study she developed mild cognitive problems- she is distractible and her manager at work has noticed a decline in her performance; so she may be at the early stages of a neurodegenerative disorder. We included her as a healthy control in the statistical analysis.

Furthermore, we did not validate the identified proteins using an alternative method, such as conventional immunoassays. We are planning to do this at a future study. Finally, the panel of candidate biomarkers in parkinsonism identified in our study does not contain several established biomarkers, such as $\alpha$-synuclein or neurofilament light chain protein[4]. More extensive sample prefractionation prior to LC-MS, to increase the CSF volume that can be analyzed while simplifying the peptide mixture loaded, will be necessary to detect such lower abundant proteins.

\section{Conclusions}

Using an unbiased approach we identified proteins differentiating parkinsonian patients from healthy controls. They mainly consist of inflammatory, neuronal and synaptic markers and they may give new clues for disease mechanisms of neurodegeneration in parkinsonism, and provide valuable biomarkers for more accurate and expedient diagnosis.

\section{Acknowledgement}

We gratefully acknowledge the support of the Swedish Research Council (5212011-4709), Emil och Wera Cornells stiftelse, Åhlens-stiftelsen, Gun och Bertil 
Stohnes stiftelse, Aina Wallström och Mary-Ann Sjöbloms stiftelse, the Leonard

Wolfson Experimental Neurology Centre, Frimurarstiftelsen, Demensförbundet, and Stiftelsen för Gamla Tjänarinnor.

\section{References}

[1] S.L. Kowal, T.M. Dall, R. Chakrabarti, M.V. Storm, A. Jain, The current and projected economic burden of Parkinson's disease in the United States, Movement disorders : official journal of the Movement Disorder Society 28(3) (2013) 311-8.

[2] S.S. O'Sullivan, L.A. Massey, D.R. Williams, L. Silveira-Moriyama, P.A. Kempster, J.L. Holton, T. Revesz, A.J. Lees, Clinical outcomes of progressive supranuclear palsy and multiple system atrophy, Brain 131(5) (2007) 13621372.

[3] A.J. Hughes, S.E. Daniel, L. Kilford, A.J. Lees, Accuracy of clinical diagnosis of idiopathic Parkinson's disease: a clinico-pathological study of 100 cases., Journal of Neurology, Neurosurgery \& Psychiatry 55(3) (1992) 181-184.

[4] N. Magdalinou, A.J. Lees, H. Zetterberg, Cerebrospinal fluid biomarkers in parkinsonian conditions: an update and future directions, Journal of neurology, neurosurgery, and psychiatry 21(3) (2014) 365-367.

[5] F. Abdi, J.F. Quinn, J. Jankovic, M. McIntosh, J.B. Leverenz, E. Peskind, R. Nixon, J. Nutt, K. Chung, C. Zabetian, A. Samii, M. Lin, S. Hattan, C. Pan, Y. Wang, J. Jin, D. Zhu, G.J. Li, Y. Liu, D. Waichunas, T.J. Montine, J. Zhang, Detection of biomarkers with a multiplex quantitative proteomic platform in cerebrospinal fluid of patients with neurodegenerative disorders., Journal of Alzheimer's disease : JAD 9(3) (2006) 293-348.

[6] R. Constantinescu, U. Andreasson, S. Li, V.N. Podust, N. Mattsson, R. Anckarsäter, H. Anckarsäter, L. Rosengren, B. Holmberg, K. Blennow, C. Wikkelsö, U. Rüetschi, H. Zetterberg, Proteomic profiling of cerebrospinal fluid in parkinsonian disorders, Parkinsonism \& related disorders 16(8) (2010) 545549.

[7] N. Ishigami, T. Tokuda, M. Ikegawa, M. Komori, T. Kasai, T. Kondo, Y. Matsuyama, T. Nirasawa, H. Thiele, K. Tashiro, M. Nakagawa, Cerebrospinal fluid proteomic patterns discriminate Parkinson's disease and multiple system atrophy, Movement disorders : official journal of the Movement Disorder Society 27(7) (2012) 851-7.

[8] S. Lehnert, S. Jesse, W. Rist, P. Steinacker, H. Soininen, S.K. Herukka, H. Tumani, M. Lenter, P. Oeckl, B. Ferger, B. Hengerer, M. Otto, iTRAQ and multiple reaction monitoring as proteomic tools for biomarker search in cerebrospinal fluid of patients with Parkinson's disease dementia, Exp Neurol 234(2) (2012) 499-505.

[9] J. Zhang, I. Sokal, E.R. Peskind, J.F. Quinn, J. Jankovic, C. Kenney, K.A. Chung, S.P. Millard, J.G. Nutt, T.J. Montine, CSF Multianalyte Profile Distinguishes Alzheimer and Parkinson Diseases, American Journal of Clinical Pathology 129(4) (2008) 526-529. 
[10] L. Dayon, A. Hainard, V. Licker, N. Turck, K. Kuhn, D.F. Hochstrasser, P.R. Burkhard, J.C. Sanchez, Relative quantification of proteins in human cerebrospinal fluids by MS/MS using 6-plex isobaric tags, Analytical chemistry 80(8) (2008) 2921-31.

[11] I. Litvan, Y. Agid, D. Calne, G. Campbell, B. Dubois, R.C. Duvoisin, C.G. Goetz, L.I. Golbe, J. Grafman, J.H. Growdon, M. Hallett, J. Jankovic, N.P. Quinn, E. Tolosa, D.S. Zee, Clinical research criteria for the diagnosis of progressive supranuclear palsy (Steele-Richardson-Olszewski syndrome): report of the NINDS-SPSP international workshop, Neurology 47(1) (1996) 1-9.

[12] R. Mathew, T.H. Bak, J.R. Hodges, Diagnostic criteria for corticobasal syndrome: a comparative study, Journal of neurology, neurosurgery, and psychiatry 83(4) (2012) 405-10.

[13] S. Gilman, G.K. Wenning, P.A. Low, D.J. Brooks, C.J. Mathias, J.Q. Trojanowski, N.W. Wood, C. Colosimo, A. Dürr, C.J. Fowler, H. Kaufmann, T. Klockgether, A. Lees, W. Poewe, N. Quinn, T. Revesz, D. Robertson, P. Sandroni, K. Seppi, M. Vidailhet, Second consensus statement on the diagnosis of multiple system atrophy., Neurology, 2008, pp. 670-676.

[14] X. Robin, N. Turck, A. Hainard, N. Tiberti, F. Lisacek, J.C. Sanchez, M. Muller, pROC: an open-source package for R and S plus to analyze and compare ROC curves, BMC bioinformatics 12 (2011).

[15] H. Wickham, ggplot2: Elegant Graphics for Data Analysis, Springer-Verlag New York2009.

[16] H.Y. Benjamini Y., A Practical and Powerful Approach to Multiple Testing, Journal of the Royal Statistical Society Series B (Methodological) 57 (1995) 289300.

[17] N.K. Magdalinou, R.W. Paterson, J.M. Schott, N.C. Fox, C. Mummery, K. Blennow, K. Bhatia, H.R. Morris, P. Giunti, T.T. Warner, R. de Silva, A.J. Lees, H. Zetterberg, A panel of nine cerebrospinal fluid biomarkers may identify patients with atypical parkinsonian syndromes, Journal of neurology, neurosurgery, and psychiatry 86(11) (2015) 1240-1247.

[18] S. Hall, Accuracy of a Panel of 5 Cerebrospinal Fluid Biomarkers in the Differential Diagnosis of Patients With Dementia and/or Parkinsonian Disorders, Archives of Neurology 69(11) (2012) 1445.

[19] M.J. Walport, Complement. First of two parts, The New England journal of medicine 344(14) (2001) 1058-66.

[20] I.L. Goldknopf, E.A. Sheta, J. Bryson, B. Folsom, C. Wilson, J. Duty, A.A. Yen, S.H. Appel, Complement $\mathrm{C} 3 \mathrm{c}$ and related protein biomarkers in amyotrophic lateral sclerosis and Parkinson's disease, Biochem Biophys Res Commun 342(4) (2006) 1034-9.

[21] Y. Furiya, M. Hirano, N. Kurumatani, T. Nakamuro, R. Matsumura, N. Futamura, S. Ueno, Alpha-1-antichymotrypsin gene polymorphism and susceptibility to multiple system atrophy (MSA), Brain research. Molecular brain research 138(2) (2005) 178-81.

[22] K. Iwase, A. Ishihara, S. Yoshimura, Y. Andoh, M. Kato, N. Seki, E. Matsumoto, T. Hiwasa, D. Muller, K. Fukunaga, M. Takiguchi, The secretogranin II gene is a signal integrator of glutamate and dopamine inputs, J Neurochem 128(2) (2014) 233-45. 
[23] G.N. Yin, H.W. Lee, J.Y. Cho, K. Suk, Neuronal pentraxin receptor in cerebrospinal fluid as a potential biomarker for neurodegenerative diseases, Brain Res 1265 (2009) 158-70.

[24] T.W. Kim, K. Wu, J.L. Xu, G. McAuliffe, R.E. Tanzi, W. Wasco, I.B. Black, Selective localization of amyloid precursor-like protein 1 in the cerebral cortex postsynaptic density, Brain research. Molecular brain research 32(1) (1995) 3644.

[25] M. Shi, J. Movius, R. Dator, P. Aro, Y. Zhao, C. Pan, X. Lin, T.K. Bammler, T. Stewart, C.P. Zabetian, E.R. Peskind, S.C. Hu, J.F. Quinn, D.R. Galasko, J. Zhang, Cerebrospinal fluid peptides as potential Parkinson disease biomarkers: a staged pipeline for discovery and validation, Molecular \& cellular proteomics : MCP (2015).

[26] P. Coskun, J. Wyrembak, S.E. Schriner, H.W. Chen, C. Marciniack, F. Laferla, D.C. Wallace, A mitochondrial etiology of Alzheimer and Parkinson disease, Biochimica et biophysica acta 1820(5) (2012) 553-64.

[27] H. Cramer, K. Rissler, N. Rosler, D. Strubel, D. Schaudt, F. Kuntzmann, Immunoreactive substance $P$ and somatostatin in the cerebrospinal fluid of senile parkinsonian patients, Eur Neurol 29(1) (1989) 1-5.

[28] H. Plun-Favreau, P.A. Lewis, J. Hardy, L.M. Martins, N.W. Wood, Cancer and neurodegeneration: between the devil and the deep blue sea, PLoS genetics 6(12) (2010) e1001257.

[29] T. Kitada, S. Asakawa, N. Hattori, H. Matsumine, Y. Yamamura, S. Minoshima, M. Yokochi, Y. Mizuno, N. Shimizu, Mutations in the parkin gene cause autosomal recessive juvenile parkinsonism, Nature 392(6676) (1998) 605-8.

[30] S. Veeriah, B.S. Taylor, S. Meng, F. Fang, E. Yilmaz, I. Vivanco, M.

Janakiraman, N. Schultz, A.J. Hanrahan, W. Pao, M. Ladanyi, C. Sander, A. Heguy, E.C. Holland, P.B. Paty, P.S. Mischel, L. Liau, T.F. Cloughesy, I.K. Mellinghoff, D.B. Solit, T.A. Chan, Somatic mutations of the Parkinson's disease-associated gene PARK2 in glioblastoma and other human malignancies, Nature genetics 42(1) (2010) 77-82.

[31] G.U. Hoglinger, N.M. Melhem, D.W. Dickson, P.M. Sleiman, L.S. Wang, L. Klei, R. Rademakers, R. de Silva, I. Litvan, D.E. Riley, J.C. van Swieten, P. Heutink, Z.K. Wszolek, R.J. Uitti, J. Vandrovcova, H.I. Hurtig, R.G. Gross, W. Maetzler, S. Goldwurm, E. Tolosa, B. Borroni, P. Pastor, P.S.P.G.S. Group, L.B. Cantwell, M.R. Han, A. Dillman, M.P. van der Brug, J.R. Gibbs, M.R. Cookson, D.G. Hernandez, A.B. Singleton, M.J. Farrer, C.E. Yu, L.I. Golbe, T. Revesz, J. Hardy, A.J. Lees, B. Devlin, H. Hakonarson, U. Muller, G.D. Schellenberg, Identification of common variants influencing risk of the tauopathy progressive supranuclear palsy, Nature genetics 43(7) (2011) 699-705.

[32] T.C. Chao, N. Hansmeier, R.U. Halden, Towards proteome standards: the use of absolute quantitation in high-throughput biomarker discovery, J Proteomics 73(8) (2010) 1641-6. 


\section{Tables}

Table 1. Demographic and clinical characteristics in the study groups. Abbreviations: avg: average, dis dur: disease duration, H\&Y score: Hoehn and Yahr score, HC: healthy controls, PD: Parkinson's disease, APS: atypical parkinsonian syndromes

Table 2: Classification of identified proteins in CSF proteome of atypical parkinsonian patients vs. healthy controls.

Abbreviations: APS: atypical parkinsonian syndrome, APP: amyloid precursor protein, FAM3C: family with sequence similarity $3 \mathrm{C}$ protein, $\boldsymbol{\uparrow} / \boldsymbol{\downarrow}$ : consistent increase/decrease of quantified tryptic peptides.

\section{Figure legends}

Figure 1. Group separation of the identified candidate biomarkers. The scale on the Y-axis is the Log2-transformed TMT-reporter ion ratios. Data are plotted for one tryptic peptide per protein. Abbreviations: HC: healthy controls, PD: Parkinson's disease, APS: atypical parkinsonian syndrome. Abbreviations: CSF: cerebrospinal fluid, TMT: tandem mass tags, LC-MS: liquid chromatography mass spectrometry, MS/MS: tandem mass spectrometry. 


\section{Supplementary Table Legends}

Table s1. Cerebrospinal fluid candidate biomarkers identified in atypical parkinsonism.

The relative difference was calculated as the median relative difference for all tryptic peptides detected for each protein in both the Discovery and Validation set. The p-values were calculated by t-test, comparing APS and HC in the Validation set. The reported p-value for each protein is the lowest $\mathrm{p}$-value for a tryptic peptide from the protein. AUC-values were obtained by ROC-curve analysis. Abbreviations: PD: Parkinson's Disease, APS: atypical parkinsonian syndrome, HC: healthy controls.

Table S2: Description of identified proteins and outline of previous CSF studies involving these proteins in neurodegenerative diseases. 